\title{
Abnormal Activation of the Social Brain Network in Children with Autism Spectrum Disorder: An fMRI Study
}

\author{
Sun-Young Kim', Uk-Su Choi' ${ }^{2}$ Sung-Yeon Park², Se-Hong Oh², Hyo-Woon Yoon ${ }^{3}$, Yun-Joo Koh ${ }^{4}$, \\ Woo-Young Im $^{5}$, Jee-In Park ${ }^{6}$, Dong-Ho Song ${ }^{6}$, Keun-Ah Cheon ${ }^{6}$, and Chang-Uk Lee ${ }^{7 凶}$ \\ 'Sungmo-Maum Psychiatric Clinic, Seoul, Republic of Korea \\ ${ }^{2}$ Neuroscience Research Institute, Gachon University of Medicine and Science, Incheon, Republic of Korea \\ ${ }^{3}$ Department of Art Therapy, Daegu Cyber University, Daegu, Republic of Korea \\ ${ }^{4}$ The Korea Institute for Children's Social Development and Rudolph Child Research Center, Seoul, Republic of Korea \\ ${ }^{5}$ Department of Psychiatry, Konyang University College of Medicine, Daejeon, Republic of Korea \\ ${ }^{6}$ Division of Child and Adolescent Psychiatry, Department of Psychiatry and Institute of Behavioral Science in Medicine, \\ Yonsei University College of Medicine, Seoul, Republic of Korea \\ ${ }^{7}$ Department of Psychiatry, Seoul St. Mary's Hospital, The Catholic University of Korea, Seoul, Republic of Korea
}

Objective The aim of this study is to investigate abnormal findings of social brain network in Korean children with autism spectrum disorder (ASD) compared with typically developing children (TDC).

Methods Functional magnetic resonance imaging (fMRI) was performed to examine brain activations during the processing of emotional faces (happy, fearful, and neutral) in 17 children with ASD, 24 TDC.

Results When emotional face stimuli were given to children with ASD, various areas of the social brain relevant to social cognition showed reduced activation. Specifically, ASD children exhibited less activation in the right amygdala (AMY), right superior temporal sulcus (STS) and right inferior frontal gyrus (IFG) than TDC group when fearful faces were shown. Activation of left insular cortex and right IFG in response to happy faces was less in the ASD group. Similar findings were also found in left superior insular gyrus and right insula in case of neutral stimulation.

Conclusion These findings suggest that children with ASD have different processing of social and emotional experience at the neural level. In other words, the deficit of social cognition in ASD could be explained by the deterioration of the capacity for visual analysis of emotional faces, the subsequent inner imitation through mirror neuron system (MNS), and the ability to transmit it to the limbic system and to process the transmitted emotion.

Psychiatry Investig 2015;12(1):37-45

Key Words Autism spectrum disorder, Social brain network, Social cognition, fMRI.

\section{INTRODUCTION}

Autism spectrum disorder (ASD) is a developmental neuro-

Received: February 24, 2014 Revised: August 18, 2014

Accepted: August 27, 2014 Available online: October 30, 2014

$\triangle$ Correspondence: Keun-Ah Cheon, MD, PhD

Division of Child and Adolescent Psychiatry, Yonsei Autism Laboratory, Department of Psychiatry and Institute of Behavioral Science in Medicine, Severance Hospital, Yonsei University College of Medicine, 50-1 Yonsei-ro, Seodaemun-gu, Seoul 120-752, Republic of Korea

Tel: +82-2-2228-1633, Fax: +82-2-313-0891, E-mail: kacheon@yuhs.ac

$\triangle$ Correspondence: Chang Uk Lee, MD, PhD

Department of Psychiatry, Seoul St. Mary's Hospital, The Catholic University of Korea, 222 Banpo-daero, Seocho-gu, Seoul 137-701, Republic of Korea Tel: +82-2-2258-6082, Fax: +82-2-536-8744, E-mail: jihan@catholic.ac.kr

(a) This is an Open Access article distributed under the terms of the Creative Commons Attribution Non-Commercial License (http://creativecommons.org/licenses/bync/3.0) which permits unrestricted non-commercial use, distribution, and reproduction in any medium, provided the original work is properly cited. biological disorder that shows symptoms of qualitative impairment of social interaction. ASD is characterized by core symptoms such as persistent deficits in social communication and social interaction across multiple contexts, and restricted repetitive patterns of behavior, interests, or activities. ${ }^{1}$ The fact that the most fundamental phenotype of ASD is the qualitative impairment of social interaction is a commonly derived clinical viewpoint, and for the past 30 years, various brain imaging studies including functional magnetic resonance imaging (fMRI) have been performed as part of the efforts to investigate the neural correlates of social deficits in ASD.

Among the results of fMRI studies, which go beyond simply evaluating a brain in terms of structure and actually evaluate the function of each brain region, enabling "in vivo investigation," one of the most persuasively replicated results is the ab- 
normality in the so-called "social brain area"2,3 The social brain area includes the superior temporal sulcus (STS) and its adjacent areas, such as the middle temporal gyrus (MTG), fusiform gyrus (FG), amygdala (AMY), medial prefrontal cortex (MPFC), and inferior frontal gyrus (IFG). It is known that the social brain area plays an important role in social cognition, which is the accumulation of cognitive processes required to comprehend and interact with others. ${ }^{4,5}$ Many previous fMRI studies have found that the ASD patients group showed hypoactivation of the social brain area compared to the healthy control group. The regions of the social brain area that showed abnormalities were different depending on the field of social cognition on which each study put a particular emphasis (e.g., emotional face processing, theory of mind, mentalizing ability, etc.) and depended on the characteristics of the task used in each study. ${ }^{3,6-8}$

To relate and understand the social deficit of ASD (i.e., clinical features) and the findings of brain imaging studies, a series of basic processes of appropriate social interaction with another person, which are qualitatively deficient for ASD patients, need to be simplified. The first step is to recognize the emotion on the facial expression of another person. The next step is to experience and share the emotional states of another person by imitating and reproducing the recognized emotion within one's own mind, which is called "empathic process." In this regard, the concept of "empathy" can be defined as "an affective state, caused by sharing of the emotions or sensory states of another person". "The next step following the empathic process is to take the perspective of another person, to understand the basic situation and intention of another person that induced a certain emotion or behavior, and to predict and perform appropriate responses. This is called "mentalizing process," and is essential for successful social interaction..$^{10,11}$

The neural correlates that are known to be related with the key processes of social interaction mentioned above (i.e., empathy and mentalization) are included in the social brain area that showed abnormality in the imaging studies of ASD patients. Specifically, emotional face perception, which is the first step in understanding the inner world of another person, is a complex visual process that accompanies the activation of anterior limbic regions (e.g., AMY) and other cortical regions (e.g., STS and cingulate) as well as the activation of FA, which is the face-selective region and is essential for the encoding of face traits and identity. ${ }^{12}$ STS is known to play a role in the visual analysis of dynamic aspects, particularly changeable aspects such as expression. In the next step, for empathy with the recognized emotion of another person, it is essential to perform a simulation process for the behavior and emotion of another person through the mirror neuron system (MNS). In other words, when we look at another person who expresses a certain emotion, we go through an inner imitation process by the activation of our MNS, and thus we can feel the emotion that another person feels, "as if we experienced the emotion".13,14 This MNS is also included in the region of the IFG of the social brain area. Furthermore, mentalizing is the ability to infer the intention behind the behavior of another person and to predict the "mental states" of another person. The regions which have been repeatedly identified as the neural correlates relevant to mentalizing based on the $\mathrm{MRRI}$ studies using various paradigms are pSTS/TPJ, temporal pols, and MPFC, ${ }^{10,11}$ which are also included in the social brain area.

The purpose of the present study was to analyze and compare the brain activation pattern of Korean children with ASD with that of typically developing children (TDC), using fMRI technique, during the performance of emotional face perception tasks. Based on the results of previous studies with ASD patients, it was hypothesized that the various regions of the social brain area of the children with ASD, who had a qualitative impairment of social interaction, would show less activation than those of TDC.

\section{METHODS}

\section{Participants}

Children with ASD and TDC were recruited from a child and adolescent psychiatric clinical group and from a community epidemiological study in the city of Goyang in South Korea. ${ }^{15}$ We initially enrolled and scanned 48 children (38 boys, 10 girls), but 5 boys and 2 girls met at least one exclusion criterion. One patient had neurofibromatosis, another had arachnoid cyst, 3 had intellectual disability, and 2 had previous seizures. Accordingly, we analyzed the data of 17 children with ASD (16 males and 1 female) and 24 children in the TDC group (17 males and 7 females). Nine of the patients with ASD were recruited from a clinical setting, and eight were enrolled from an ongoing epidemiological study. Eight of the patients with ASD from the clinic were being treated with methylphenidate for 27 to 170 days, and one was treated with atomoxetine for 27 days. One boy taking methylphenidate was also treated with valproic acid for 68 days prior to scanning. All participants from the epidemiological sample were medication-naive.

All TDC were recruited from a community epidemiological study ${ }^{15}$ and were screened by two child and adolescent psychiatrists with assessments including an intensive interview and a psychological evaluation. Out of 30 children recruited, 6 were found to have $\operatorname{ADHD}(n=3)$, intellectual disability $(n=2)$, and arachnoid cyst $(n=1)$ during the brain imaging. Therefore, 24 TDC were included in the final analysis.

There were no significant differences in age (ASD; 10.89 \pm 
2.06, TDC; 10.18 \pm 2.04 ) and intelligence (ASD; 112.67 \pm 12.73 , TDC; $112.54 \pm 11.62)$ between the two groups. But there were a relative deviation in sex ratio (ASD; 16 males and 1 female, TDC; 17 males and 7 females) between the two groups. Table 1 summarizes the demographic data of the two groups.

In the first selection step for diagnosing ASD, the Autism Spectrum Screening Questionnaire (ASSQ) ${ }^{16}$ (Korean version is in the process of standardization) was used (cut off; 13 point). In the next step, using the Autism Diagnosis InterviewRevised-Korean version (ADI-R) ${ }^{17}$ and Autism Diagnostic Observation Schedule-Korean version (ADOS), ${ }^{18}$ two certified raters who were skilled at using these tools, made a final diagnosis. Among the children diagnosed with ASD, patients from the age of 6-12 with an intelligence quotient of more than 80 (Korean Educational Development Institute-Wechsler Intelligence Scale for Children-revised-III; KEDI-WISC were used) were selected as the final subjects, and children without any child psychiatric disorder were matched as the normal control group. Exclusion criteria for the ASD group were 1) a past or present history of brain damage or convulsive disorder; 2) intellectual disability or specific language impairment; and 3) comorbid child and adolescent psychiatric disorders based on DSM-IV-TR.

The Schedule for Affective Disorders and Schizophrenia for Children-Present and Lifetime Version-Korean Version (KSADS-PL-K $)^{19}$ which is a semi-structured interview schedule, was performed on all the research participants, and those who were diagnosed with comorbid psychiatric disorders were excluded from the study.

The Social Responsiveness Scale (SRS)-Korean version which is a 65 -item rating scale that quantifies the presence and severity of autism symptoms, was also performed on all the

Table 1. Demographic and neuropsychological data of the subjects

\begin{tabular}{|c|c|c|c|}
\hline & $\operatorname{ASD}(\mathrm{N}=17)$, mean $\pm \mathrm{SD}$ & $\mathrm{TDC}(\mathrm{N}=24)$, mean $\pm \mathrm{SD}$ & $\mathrm{p}$ \\
\hline \multicolumn{4}{|l|}{ Sex } \\
\hline Female/Male (\%) & $1 / 16(6.25)$ & $7 / 17(41.17)$ & 0.064 \\
\hline Age & $10.89 \pm 2.06$ & $10.18 \pm 2.04$ & 0.250 \\
\hline \multicolumn{4}{|l|}{ IQ } \\
\hline Verbal IQ & $115.13 \pm 10.13$ & $111.33 \pm 12.90$ & 0.337 \\
\hline Performance IQ & $106.40 \pm 16.21$ & $110.88 \pm 11.25$ & 0.538 \\
\hline Total IQ & $112.67 \pm 12.73$ & $112.54 \pm 11.62$ & 0.717 \\
\hline \multicolumn{4}{|c|}{ Continuous Performance Test (visual) T-scores } \\
\hline Omission error & $53.00 \pm 11.13$ & $49.00 \pm 9.17$ & 0.078 \\
\hline Commission error & $61.33 \pm 20.80$ & $49.95 \pm 8.35$ & 0.059 \\
\hline Reaction time & $52.47 \pm 17.23$ & $54.33 \pm 10.20$ & 0.891 \\
\hline Reaction time SD & $68.13 \pm 26.54$ & $54.33 \pm 9.72$ & $0.0044^{*}$ \\
\hline $\mathrm{HC}(\mathrm{cm})$ & $55.24 \pm 1.25$ & $55.07 \pm 3.10$ & 0.932 \\
\hline \multicolumn{4}{|l|}{ Handedness } \\
\hline Left/Right (\%) & $1 / 16(6.25)$ & $1 / 23(4.16)$ & 0.802 \\
\hline Total SRS score & $75.00 \pm 41.01$ & $26.73 \pm 18.48$ & $0.002^{*}$ \\
\hline \multicolumn{4}{|l|}{ ADI-R-K } \\
\hline Social & $16.31 \pm 6.62$ & - & - \\
\hline Communication & $12.31 \pm 4.22$ & - & - \\
\hline Repetitive behavior & $4.50 \pm 2.50$ & - & - \\
\hline Birth threshold & $2.19 \pm 1.42$ & - & - \\
\hline \multicolumn{4}{|l|}{ ADI-R-K } \\
\hline Communication & $3.06 \pm 1.12$ & - & - \\
\hline Social & $8.75 \pm 2.17$ & - & - \\
\hline Creativity & $1.00 \pm 0.63$ & - & - \\
\hline Restrictive behavior & $2.06 \pm 1.12$ & - & - \\
\hline
\end{tabular}

The statistical analyses were done by independent $\mathrm{t}$-test. ${ }^{*} \mathrm{p}<0.05$. ASD: autism spectrum disorders, ADI-R-K: autism diagnosis interview-revised-korean version, ADOS-K: Autism Diagnostic Observation Schedule-Korean version, HC: head circumference, SD: standard deviation, SRS: Social Responsiveness Scale, TDC: typically developing children 
participants. The SRS makes a singular scale score that serves as an index of severity of social deficits in the ASD. ${ }^{20}$ For the SRS scores, the ASD group (75.00 \pm 41.01$)$ showed significantly higher value than the control group $(26.73 \pm 18.48)$ (Table 1$)$.

To quantitatively measure inattention (omission errors) and impulsivity (commission errors), the Continuous Performance Test (CPT) was performed on all the participants. For the CPT, the standard deviation of the visual reaction time was significantly higher in the ASD group $(68.13 \pm 26.54)$ than in the control group (54.33 \pm 9.72 ) (Table 1).

The present study was approved by the institutional review boards for research with human subjects at the University Hospital where the present study was performed and at the Gachon Neuroscience Research Institute in Incheon, South Korea, where all the subjects were scanned. All the subjects and their parents were given a full description of the study, and gave prior written informed assent and consent.

\section{Experimental paradigm \& data acquisition}

Brain $\mathrm{fMRI}$ images were acquired using the 1.5 Tesla (Avanto, Siemens) instrument at the Neuroscience Research Institute, Gachon University of Medicine and Science.

In terms of the paradigm used in the present study, three categories of emotional face pictures (happy, fearful, and neutral) were shown to the subjects, and the brain activities of the subjects were observed and compared between the ASD group and the control group, while they were passively observing the pictures. The emotional face stimuli for each category were presented as a 'block' unit. In a block, each emotional face picture was shown for 2 seconds (a blank screen was shown for 1 second immediately after the picture), and six emotional face pictures were shown per block (Figure 1).

The participants were instructed to distinguish the gender of the faces rather than identify the expression of the faces, and the emotional face stimuli used in the experiment were taken from the standardized data bank of the International Affective Picture System (IAPS). ${ }^{21}$

\section{fMRI data analysis}

The functional data and anatomical data were analyzed using the Brain Voyager QX software (Brain Innovation, Maastricht, The Netherlands). The preprocessing of the functional data included slice scan time correction, 3D-motion correction, linear trend removal, and high-pass filtering. After the preprocessing steps, the data were co-registered with the anatomical data, which were transformed to Talairach space. The data were analyzed using random-effect GLM and ANOVA analysis, which were incorporated into BrainVoyager QX. All the data were beta values in each voxel. After the random-effect GLM analysis, all the extracted data were analyzed using two-way ANOVA with stimulus factor (happy, fearful and neutral face) and group factor (ASD and TDC). In the whole-brain analyses, specific clusters were found according to the main effects and interactions.

The threshold of activation in all clusters was $\mathrm{p}<0.005$ for a spatial extent of contiguous 30 voxels and was uncorrected for multiple comparisons.

We also calculated Pearson correlations between BOLD responses during processing of emotional faces and the SRS total score using SPSS version 19.0. Statistical significance thresholds were set at $\mathrm{p}<0.05$.

\section{RESULTS}

ANOVAs revealed that for the fearful faces, the ASD group showed lower activation of the right AMY ( $p=0.0067$, uncorrected), right STS ( $\mathrm{p}=0.0003$, uncorrected), and right IFG $(\mathrm{p}=0.0119$, uncorrected) areas than the TDC group. For the

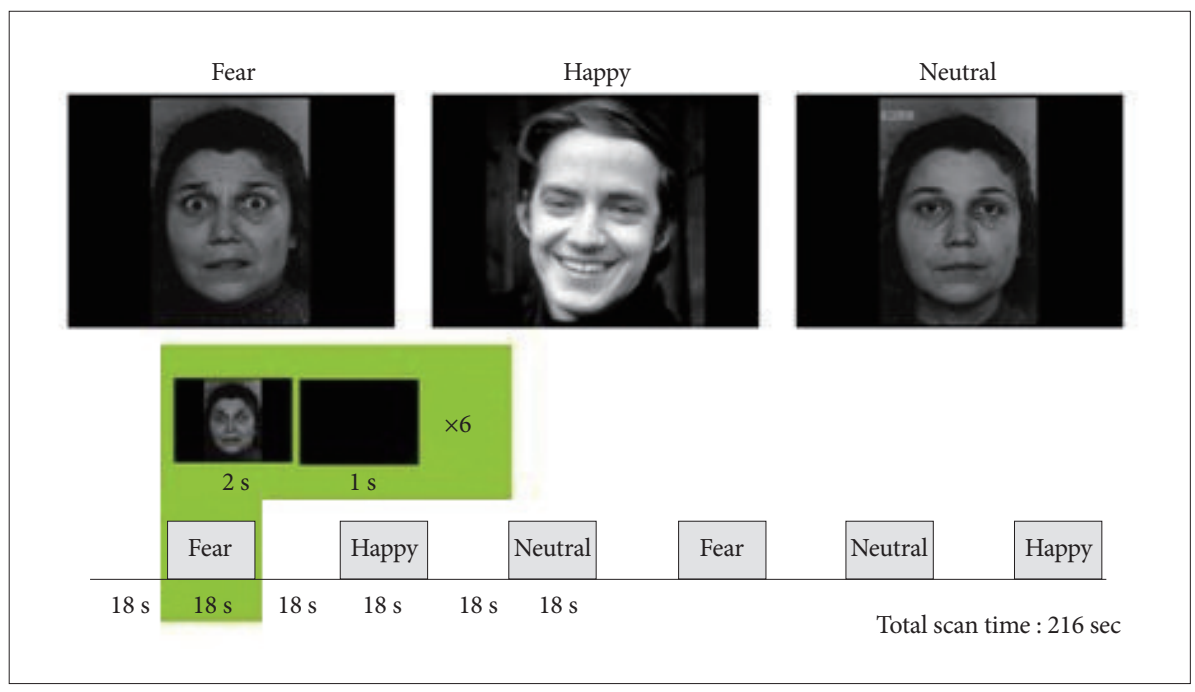

Figure 1. Paradigm design and examples of the stimuli used in the emotional face perception task. The emotional face stimuli for each category (happy, fear, and neutral) were presented as a block unit. In a block, each emotional face picture was shown for 2 seconds (a blank screen was shown for 1 second immediately after the picture), and six emotional face pictures were shown per block. 
Table 2. Regions showing significant differences in BOLD activations between the ASD and TDC groups (uncorrected, $p<0.01$ )

\begin{tabular}{|c|c|c|c|c|c|c|c|}
\hline $\begin{array}{c}\text { Group } \\
\text { comparison }\end{array}$ & Condition & Brain region & $\begin{array}{c}\text { Brodmann } \\
\text { area }\end{array}$ & $\begin{array}{c}\text { Talairach } \\
\text { coordinates }\end{array}$ & F-value & $\mathrm{p}$ & Cluster size \\
\hline \multirow[t]{9}{*}{$\mathrm{ASD}<\mathrm{TDC}$} & \multirow[t]{3}{*}{ Fearful face } & R. Amygdala & $\mathrm{N} / \mathrm{A}$ & $21,-8,-20$ & 8.2 & 0.0067 & 33 \\
\hline & & R. Superior temporal gyrus & 22 & $54,-20,-1$ & 15.4 & 0.0003 & 87 \\
\hline & & R. Inferior frontal gyrus & 45 & $53,21,22$ & 7.0 & 0.0119 & 88 \\
\hline & \multirow[t]{3}{*}{ Happy face } & L. Insular cortex & N/A & $-35,30,-2$ & 12.1 & 0.0013 & 90 \\
\hline & & L. Middle frontal gyrus & 6 & $-30,-12,39$ & 16.5 & 0.0002 & 68 \\
\hline & & R. Inferior frontal gyrus & 46 & $44,-32,19$ & 24.9 & 0.00001 & 49 \\
\hline & \multirow[t]{3}{*}{ Neutral face } & L. Post central gyrus & 40 & $-53,-22,21$ & 9.0 & 0.0047 & 42 \\
\hline & & L. Superior insular gyrus & 13 & $-37,-3,14$ & 11.6 & 0.0016 & 49 \\
\hline & & R. Insula & N/A & $-25,25,13$ & 15.8 & 0.0003 & 83 \\
\hline \multirow[t]{8}{*}{ ASD $>$ TDC } & \multirow[t]{3}{*}{ Fearful Face } & Dorsal posterior cingulate gyrus & 30 & $-3,-49,22$ & 10.5 & 0.0024 & 383 \\
\hline & & L. Superior frontal gyrus & 10 & $-13,60,9$ & 21.1 & 0.00005 & 347 \\
\hline & & L. Inferior frontal sulcus & 9 & $-53,0,28$ & 23.9 & 0.00002 & 434 \\
\hline & \multirow[t]{2}{*}{ Happy Face } & R. Occipital gyrus & 17 & $14,-93,6$ & 16.3 & 0.0002 & 426 \\
\hline & & L. Superior temporal gyrus & 22 & $-58,27,5$ & 17.8 & 0.0001 & 142 \\
\hline & \multirow[t]{3}{*}{ Neutral face } & R. Parietal lobe & N/A & $37,-52,32$ & 15.8 & 0.0003 & 184 \\
\hline & & R. Precuneus & 7 & $6,-68,34$ & 14.6 & 0.0005 & 167 \\
\hline & & R. Middle frontal gyrus & 8 & $25,9,35$ & 13.0 & 0.0009 & 197 \\
\hline
\end{tabular}

The statistical analyses were done by random effect GLM and ANOVA analysis. ASD: autism spectrum disorder, ASD $<$ TDC: the regions in which the ASD group shows lower activation than the TDC group, ASD > TDC: the regions in which the ASD group shows higher activation than the TDC group, L: left, N/A: not applicable, R: right, TDC: typically developing children

happy faces, the ASD group showed reduced activation of the left insular cortex $(\mathrm{p}=0.0013$, uncorrected), left MFG ( $\mathrm{p}=$ 0.0002 , uncorrected), and right IFG ( $\mathrm{p}=0.00001$, uncorrected) areas than the TDC group; and for the neutral faces, the ASD group showed lower activation of the left posterior central gyrus $(\mathrm{p}=0.0047$, uncorrected), left superior insular gyrus $(\mathrm{p}=$ 0.0016 , uncorrected), and right insular ( $\mathrm{p}=0.0003$, uncorrected) areas than the TDC group.

On the other hand, there were areas in which the ASD group showed higher activation than the TDC group. For the fearful faces, the ASD group showed higher activation of the dorsal posterior cingulate gyrus ( $\mathrm{p}=0.0024$, uncorrected), left superior frontal gyrus ( $\mathrm{p}=0.00005$, uncorrected), and left inferior frontal sulcus ( $\mathrm{p}=0.00002$, uncorrected) areas than the TDC group; and for the happy faces, the ASD group showed higher activation of the right occipital gyrus ( $p=0.0002$, uncorrected) and left superior temporal gyrus ( $\mathrm{p}=0.0001$, uncorrected) areas than the TDC group. For the neutral faces, the ASD group showed higher activation of the right parietal lobe ( $\mathrm{p}=0.0003$, uncorrected), right precuneus $(\mathrm{p}=0.0005$, uncorrected), and right middle frontal gyrus ( $\mathrm{p}=0.0009$, uncorrected) areas than the TDC group. Table 2 and Figure 2 summarize the brain activation for each emotional face, and the comparison of the BOLD response between the two groups.

In correlation analyses with the psychosocial scales, BOLD response of ROI was not significantly correlated with total SRS score in subjects with ASD.

\section{DISCUSSION}

In the present study, the results of several previous studies relevant to the abnormal activation of social brain in ASD were replicated significantly. The so-called "social brain network" refers to the STS or its adjacent regions such as the MTG, FG, AMY, MPFC, and IFG. It is known that this social brain network plays a pivotal role in social interaction.

As mentioned earlier, the core of the processes of social interaction with another person is the empathic ability, by which one recognizes the emotion of another person and feels the emotion that another person feels by imitating and reproducing the recognized emotion within one's own mind. Empathy is "the process of purposely putting one-self in another person's shoes in order to understand that person's thoughts and feelings".22 Although we recognize the distinction between ourselves and the other person, we momentarily try to create the other person's internal state within ourselves to better understand that perspective. ${ }^{22}$ The regions relevant to this simulation mechanism are AMY, MPFC, and insular; and previous studies have suggested that the dysfunction of this simulation mechanism was found in ASD. ${ }^{23}$ 

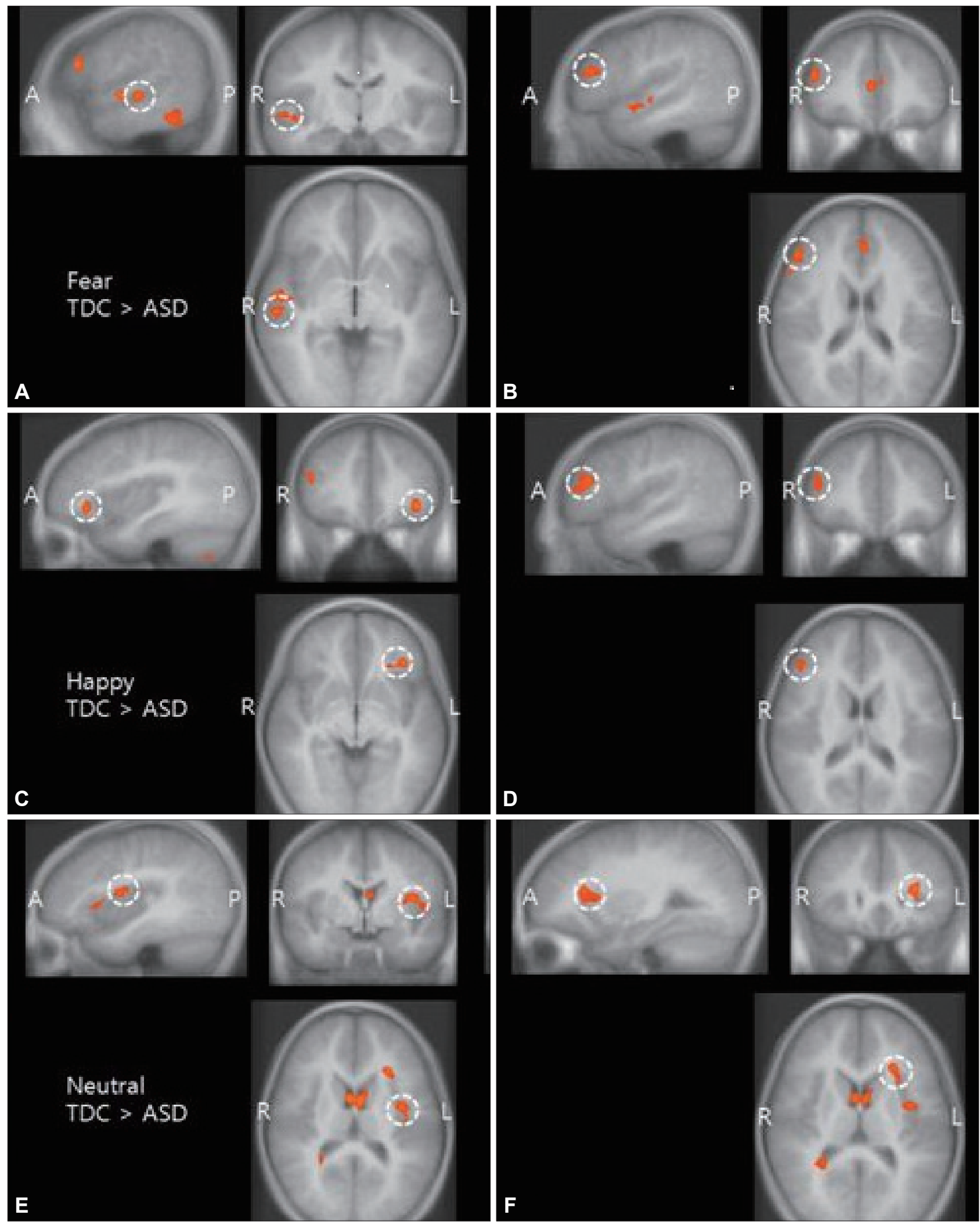

Figure 2. Main effect of emotional face perception (A and B: fearful stimuli, C and D: happy stimuli, E and F: neutral stimuli). Reduced activities were observed in several parts of the social brain network in the ASD group compared with the TDC group while pictures of emotional faces were shown (uncorrected, $p<0.01$ ). Brain regions indicated are right superior temporal sulcus (STS) (A), right inferior frontal gyrus (IFG) (B); left insular cortex (C); right inferior frontal gyrus (IFG) (D); left superior insular sulcus (E); left superior insular sulcus (F). ASD: autism spectrum disorder, TDC: typically developing children, A: anterior, P: posterior, R: right, L: left. 
When examined based on the sequence of the process and the neural correlates, STS is related with the visual analysis of dynamic aspects, and is particularly involved in changeable aspects such as facial expressions. In other words, STS is the region that is critical for action representation, and plays a role in coding the early visual description of action and sending it to the MNS. ${ }^{13}$ The results of the present study indicated that for the fearful face stimulus, the ASD group showed lower activation of STS than the TDC group. This suggests the possibility that the visual analysis and representation of the expression of fearful emotion were particularly deficient for the ASD group.

The neural correlates that are related with the "inner imitation" of the information on the observed emotion of another person within one's own mind are the specific neuronal populations called "mirror neurons," and it is known that IFG is one of the regions that contain the mirror neurons. ${ }^{24}$ Many previous studies have suggested that the dysfunction of this IFG (i.e., the dysfunction of empathic processes, including inner imitation) is one of the fundamental deficits in ASD. ${ }^{25-27}$ In particular, Dapretto et al. ${ }^{27}$ performed an fMRI study of children with ASD, which was related with MNS. They found that part of the inferior frontal gyrus (a region in MNS) of the children with ASD showed reduced brain activation during the imitation and observation of emotional face stimuli, and that the degree was negatively correlated with the severity of autism spectrum disorder. The results of the present study also indicated that the ASD group showed hypo-activation of the IFG region compared to the TDC group when they were exposed to the emotional face stimuli (e.g., fearful and happy faces). This supports the results of previous studies that reported the abnormality of MNS in ASD.

The insular region plays a role in connecting to the limbic system (i.e., emotional center) so that one can feel the emotion of another person as if it was one's own emotion, through the inner imitation occurring in MNS. Anatomically, the insular region is connected to both MNS and the limbic system. ${ }^{28}$ The results of the present study indicated that for the happy and neutral face stimuli, the ASD group showed reduced activation of the insular area compared to the TDC group. The insular area is considered a central brain region in sensorimotor, visceral, interoceptive processing, and emotional awareness of self and others, interacting with the limbic, somatosensory, and motor regions. ${ }^{29}$ As physical sensation is the basis of emotion, interoception by the insular region is an important means of knowing "how I feel."30 Also, it has been theorized that insular plays a pivotal role in "the ability to feel the emotion of another person" by transmitting the sensorimotor impressions (i.e., visual sense, auditory sense, and feeling) of another person's emotional behavior to AMY, which is the limbic system that induces emotional response. ${ }^{31}$ Impaired understanding of others' sensations and emotions as well as abnormal experience of their own emotions and sensations is frequently reported in individuals with $\mathrm{ASD} .^{32}$ In addition, several neuroimaging studies have found that the reduced emotional awareness of self and others observed in ASD patients was related with the functional impairment of insular and AMY.32,33 Therefore, the hypo-activation or reduced activation of the insular area in the ASD group in response to the emotional faces, which was one of the results of the present study, supports these previous studies.

Also, AMY, which is the main area of the social brain, encompasses parts of the limbic system that is called the "emotional brain," and the neural basis that processes feelings and constitutes the emotional self. AMY is involved in the processing of emotions, especially fear. ${ }^{34}$ As has been commonly replicated in the previous facial affect processing studies of ASD patients, ${ }^{6,8}$ the results of the present study indicated that for the fearful face stimulus, the activation of amygdala was significantly reduced in the ASD group. This reduced activation of AMY in the ASD group supports the 'amygdala theory of autism,' which is the representative model of the pathophysiology of ASD (Abnormality occurs in the development of AMY at the initial stage of brain development, and it causes a problem in the cortex that mediates social cognition, especially the FFA of the ventral temporal lobe. This then induces the deficit of social perception)..$^{35}$

As another finding of the present study, it was found that for the neutral face stimulus, Rt. precuneus showed enhanced activation in the ASD group. This result was also reported in the previous facial affect processing studies of children and adolescents with ASD, and it could be explained that the precuneus, which is modulated depending on attentional demands, was compensatorily activated instead of the activation of FG or AMY. $^{8}$

Various visual areas (e.g., fusiform gyrus, inferior and middle occipital gyri, lingual gyrus, etc.) are involved in the processing of emotional faces. The results of the present study indicated that the ASD group did not show reduced activation of these visual areas compared to the control group, and in the case of the happy face stimulus, the ASD group showed rather enhanced activation of Rt. occipital gyri compared to the control group. This could be interpreted as indicating that although visual perception and analysis are essential for successful social interaction, the subsequent processes such as inner imitation, emotional processing, and the interpretation of the intention behind the behavior of another person are also critical.

In the case of the response to the fearful face stimulus, the reduced activation of the "right" AMY, STS, and IFG was observed in the ASD group. According to the "right-hemisphere hypothesis," the two cerebral hemispheres are specialized in 
different ways with respect to the processing of emotion. In other words, the right hemisphere is uniquely skilled at processing emotion, and the left hemisphere has a supporting role in emotional perception. ${ }^{36}$ Also, it seems that the tasks relevant to emotion are divided between the two hemispheres of the brain, with the right hemisphere specialized in the perception of negative or avoidance-related emotions, while the left hemisphere is activated by the emotions from positive experience. ${ }^{37,38}$ Therefore, the result of the present study, in which the ASD group showed abnormal activation mostly in the right social brain area in response to the fearful face stimulus, could be partly explained.

The present study had several limitations. First, in the present study, the experiment was performed using static facial expression pictures, but this is somewhat different from real-life situations. As dynamic facial expressions can provide more appropriate stimuli related with real-life social interaction, they need to be incorporated into future studies through the use of video clips, etc. Second, there is a possibility that appropriate emotion processing could not be induced because Asian children were unfamiliar with the facial expressions of Westerners, which had been adopted from the data bank of the International Affective Picture System (IAPS). ${ }^{21}$ For instance, the cause of the enhanced activation of the insular area in the normal control group compared to the ASD group in response to the neutral facial stimulus could be interpreted differently. According to the normal atlas study of facial emotion processing, it was reported that fear is related with the activation of amygdala, anger is related with the activation of ventral basal ganglia, and disgust is related with the activation of insula. ${ }^{39}$ It is possible that the activation of a specific brain area was induced because the Korean subjects, who were unfamiliar with the emotional faces of Westerners, felt emotion from the neutral facial stimulus of the present study. In the future, emotional face pictures with which Korean children are familiar need to be standardized for the study of emotion recognition, and the stimuli of basic emotions such as disgust, sadness, and anger need to be included in addition to the fear/happy/neutral stimuli. Third, a limitation of the present study was that the number of research participants was small. Also, the children with ASD who participated in the present study consisted of relatively high functioning children with ASD whose IQs are above 80 (115.13 on average), and as such it will be difficult to apply the results of the present study to lower functioning children. In addition, several findings that are inconsistent with each other or with the previous study results need to be examined. In the present study, the difference in the activation of the FFA region, which is one of the most commonly replicated results in the facial emotion recognition task for ASD, was not observed; and inconsistent results were obtained, where the activation of Rt. IFG was reduced in the ASD group in response to the fearful face stimulus, while the activation of Lt. IFG was rather enhanced in the ASD group, although it was attempted to explain this based on the characteristics of the left and right hemispheres regarding emotion ("right-hemisphere hypothesis"). Furthermore, the results of the happy and neutral face stimuli could not be explained based only on the characteristics of the left and right hemispheres. The results of the present study could explain the qualitative impairment of social interaction in ASD. However, the symptoms of ASD such as the restricted repetitive patterns of behavior, interests, or activities, and the disgust and hypersensitivity related to specific interoceptive cues cannot be explained only by the abnormality of the social brain area. Lastly, the limitation of the present study related with the analysis of the fMRI data was that the false discovery rate (FDR) correction was not applied to the images obtained from the fMRI. No statistically significant difference was noted between ASD group and TDC group in BOLD activations after applying a FDR correction. Small sample size could have caused our result to lose its significance after the FDR correction. It is also possible that the fMRI task used in the present study was not able to provoke significant difference in brain activity involving emotion processing. In addition to those possibilities, our result of activated clusters still could have significance. Previous study reported that the threshold of $\mathrm{p}<0.005$ with contiguous 10 voxels showed similar statistical power compared to an FDR of $0.05 .^{40}$ The purpose of our threshold of activated clusters was to reduce Type II errors which could increase the possibility of finding meaningful brain regions.

In conclusion, it was found that when emotional face stimuli were given to children with ASD during the experiment, the various areas of the social brain relevant to social cognition showed reduced activation. In other words, the deficit of social cognition in ASD could be explained by the deterioration of the capacity for visual analysis of emotional faces, the subsequent inner imitation through MNS, and the ability to transmit it to the limbic system and to process the transmitted emotion. In the future, the fMRI study using more various types of emotional task and much larger ASD sample will be needed for identifying neurobiological correlates of social deficit in ASD subjects.

\section{Acknowledgments}

This research was supported by a grant from the Korean Health Technology R\&D Project, Ministry of health \& welfare, Republic of Korea (HI12C0021, HI12C0245).

This study was supported by a faculty research grant of Yonsei University College of Medicine for 2010 (6-2010 0139). 


\section{REFERENCES}

1. American Psychiatric Association. Diagnostic and Statistical Manual of Mental Disorders, DSM-V. Washington, DC: American Psychiatric Association; 2013.

2. Philip RC, Dauvermann MR, Whalley HC, Baynham K, Lawrie SM, Stanfield AC. A systematic review and meta-analysis of the fMRI investigation of autism spectrum disorders. Neurosci Biobehav Rev 2012; 36:901-942.

3. Sato W, Toichi M, Uono S, Kochiyama T. Impaired social brain network for processing dynamic facial expressions in autism spectrum disorders. BMC Neurosci 2012;13:99.

4. Burnett S, Sebastian C, Cohen Kadosh K, Blakemore SJ. The social brain in adolescence: evidence from functional magnetic resonance imaging and behavioural studies. Neurosci Biobehav Rev 2011;35:16541664.

5. Johnson MH, Griffin R, Csibra G, Halit H, Farroni T, de Haan M, et al. The emergence of the social brain network: evidence from typical and atypical development. Dev Psychopathol 2005;17:599-619.

6. Ashwin C, Baron-Cohen S, Wheelwright S, O’Riordan M, Bullmore ET. Differential activation of the amygdala and the 'social brain' during fearful face-processing in Asperger Syndrome. Neuropsychologia 2007; 45:2-14.

7. Wang AT, Lee SS, Sigman M, Dapretto M. Reading affect in the face and voice: neural correlates of interpreting communicative intent in children and adolescents with autism spectrum disorders. Arch Gen Psychiatry 2007;64:698-708.

8. Wang AT, Dapretto M, Hariri AR, Sigman M, Bookheimer SY. Neural correlates of facial affect processing in children and adolescents with autism spectrum disorder. J Am Acad Child Adolesc Psychiatry 2004; 43:481-490.

9. Hein G, Singer T. I feel how you feel but not always: the empathic brain and its modulation. Curr Opin Neurobiol 2008;18:153-158.

10. Frith U, Frith CD. Development and neurophysiology of mentalizing. Philos Trans R Soc Lond B Biol Sci 2003;358:459-473.

11. Frith CD, Frith U. The neural basis of mentalizing. Neuron 2006;50: 531-534.

12. Vuilleumier P, Pourtois G. Distributed and interactive brain mechanisms during emotion face perception: evidence from functional neuroimaging. Neuropsychologia 2007;45:174-194.

13. Carr L, Iacoboni M, Dubeau MC, Mazziotta JC, Lenzi GL. Neural mechanisms of empathy in humans: a relay from neural systems for imitation to limbic areas. Proc Natl Acad Sci U S A 2003;100:5497-5502.

14. Rizzolatti G, Fogassi L, Gallese V. Neurophysiological mechanisms underlying the understanding and imitation of action. Nat Rev Neurosci 2001;2:661-670.

15. Kim YS, Leventhal BL, Koh YJ, Fombonne E, Laska E, Lim EC, et al. Prevalence of autism spectrum disorders in a total population sample. Am J Psychiatry 2011;168:904-912.

16. Ehlers S, Gillberg C, Wing L. A screening questionnaire for Asperger syndrome and other high-functioning autism spectrum disorders in school age children. J Autism Dev Disord 1999;29:129-141.

17. Lord C, Rutter M, Le Couteur A. Autism Diagnostic Interview-Revised: a revised version of a diagnostic interview for caregivers of individuals with possible pervasive developmental disorders. J Autism Dev Disord 1994;24:659-685.

18. Lord C, Risi S, Lambrecht L, Cook EH Jr, Leventhal BL, DiLavore PC, et al. The autism diagnostic observation schedule-generic: a standard measure of social and communication deficits associated with the spectrum of autism. J Autism Dev Disord 2000;30:205-223.

19. Kim YS, Cheon KA, Kim BN, Chang SA, Yoo HJ, Kim JW, et al. The reliability and validity of Kiddie-Schedule for Affective Disorders and Schizophrenia-Present and Lifetime Version-Korean version (K-SADS-
PL-K). Yonsei Med J 2004;45:81-89.

20. Constantino JN, Davis SA, Todd RD, Schindler MK, Gross MM, Brophy SL, et al. Validation of a brief quantitative measure of autistic traits: comparison of the social responsiveness scale with the autism diagnostic interview-revised. J Autism Dev Disord 2003;33:427-433.

21. Lang PJ, Bradely MM, Cuthbert BN. International Affective Picture System (IAPS): affective ratings of pictures and instruction manual. Technical Report A-8. Gainesville: University of Florida; 2008.

22. Gazzaniga MS, Ivry RB, Mangun GR. Cognitive Neuroscience: The Biology of the Mind. New York: Norton \& Company; 2008.

23. Oberman LM, Ramachandran VS. The simulating social mind: the role of the mirror neuron system and simulation in the social and communicative deficits of autism spectrum disorders. Psychol Bull 2007; 133:310-327.

24. Iacoboni M, Woods RP, Brass M, Bekkering H, Mazziotta JC, Rizzolatti G. Cortical mechanisms of human imitation. Science 1999;286:25262528.

25. Williams JH, Whiten A, Suddendorf T, Perrett DI. Imitation, mirror neurons and autism. Neurosci Biobehav Rev 2001;25:287-295.

26. Ramachandran VS, Oberman LM. Broken mirrors: a theory of autism. Sci Am 2006;295:62-69.

27. Dapretto M, Davies MS, Pfeifer JH, Scott AA, Sigman M, Bookheimer SY, et al. Understanding emotions in others: mirror neuron dysfunction in children with autism spectrum disorders. Nat Neurosci 2006; 9:28-30.

28. Williams JH, Waiter GD, Gilchrist A, Perrett DI, Murray AD, Whiten A. Neural mechanisms of imitation and 'mirror neuron' functioning in autistic spectrum disorder. Neuropsychologia 2006;44:610-621.

29. Craig AD. How do you feel--now? The anterior insula and human awareness. Nat Rev Neurosci 2009;10:59-70.

30. Pollatos O, Gramann K, Schandry R. Neural systems connecting interoceptive awareness and feelings. Hum Brain Mapp 2007;28:9-18.

31. Critchley HD, Wiens S, Rotshtein P, Ohman A, Dolan RJ. Neural systems supporting interoceptive awareness. Nat Neurosci 2004;7:189-195.

32. Ebisch SJ, Gallese V, Willems RM, Mantini D, Groen WB, Romani GL, et al. Altered intrinsic functional connectivity of anterior and posterior insula regions in high-functioning participants with autism spectrum disorder. Hum Brain Mapp 2011;32:1013-1028.

33. Silani G, Bird G, Brindley R, Singer T, Frith C, Frith U. Levels of emotional awareness and autism: an fMRI study. Soc Neurosci 2008;3:97112.

34. Gur RC, Schroeder L, Turner T, McGrath C, Chan RM, Turetsky BI, et al. Brain activation during facial emotion processing. Neuroimage 2002; 16:651-662.

35. Schultz RT. Developmental deficits in social perception in autism: the role of the amygdala and fusiform face area. Int J Dev Neurosci 2005; 23:125-141.

36. Smith SD, Bulman-Fleming MB. A hemispheric asymmetry for the unconscious perception of emotion. Brain Cogn 2004;55:452-457.

37. Davidson RJ. Cerebral Asymmetries, Emotion, and Affective Style. Cambridge: MIT Press; 1995.

38. Smith SD, Bulman-Fleming MB. An examination of the right-hemisphere hypothesis of the lateralization of emotion. Brain Cogn 2005; 57:210-213.

39. Fusar-Poli P, Placentino A, Carletti F, Landi P, Allen P, Surguladze S, et al. Functional atlas of emotional faces processing: a voxel-based metaanalysis of 105 functional magnetic resonance imaging studies. J Psychiatry Neurosci 2009;34:418-432.

40. Lieberman MD, Cuningham WA. Type I and Type II error concerns in fMRI research: re-balancing the scale. Soc Cogn Affect Neurosci 2009; 4:423-428. 